01,02

\title{
Измерение параметров сверхпроводникового потокового кубита в квазидисперсионном режиме
}

\author{
(С) И.Л. Новиков ${ }^{1}$, Б.И. Иванов ${ }^{\llbracket, 1}$, А.Н. Султанов ${ }^{1}$, Я.С. Гринберг ${ }^{1}$, Е.В. Ильичев ${ }^{1,2}$ \\ ${ }^{1}$ Новосибирский государственный технический университет, \\ Новосибирск, Россия \\ ${ }^{2}$ Leibniz Institute of Photonic Technology, \\ Jena, Germany \\ ๆ E-mail: b.ivanov@corp.nstu.ru
}

\begin{abstract}
Сообщается о результатах исследования сверхпроводникового потокового квантового бита (кубита) в квазидисперсионном режиме, когда частота пробного сигнала меньше, но тем не менее близка к частоте возбуждения кубита. В этом режиме, в отличие от известных экспериментов, взаимодействие кубита с волноводом приводит не только к смещению резонансной частоты, что характерно для дисперсионного режима, но также к заметному уширению резонансной линии, обусловленному спонтанным излучением кубита. Это позволяет на основе анализа амплитудно-частотной характеристики сигнала прохождения определить при одночастотном возбуждении характерные параметры кубита, индуктивно связанного с копланарным резонатором.
\end{abstract}

Работа выполнена при финансовой поддержке Российского научного фонда в рамках проекта № 16-19-10069.

\section{1. Введение}

В настоящее время, благодаря развитию тонкопленочных технологий, достигнут существенный прогресс в создании широкого класса твердотельных гибридных структур, содержащих на одном чипе одну или несколько макроскопических двухуровневых систем, встроенных в высокодобротный микроволновой резонатор [1]. Такие системы получили в литературе название искусственных атомов, или кубитов, поскольку при определенных условиях они проявляют квантовые свойства, присущие обычным атомам [2].

Настоящая работа посвящена исследованию одного класса таких искусственных атомов - сверхпроводниковому потоковому кубиту (СПК) [3]. Такой кубит представляет собой сверхпроводниковую петлю, замкнутую тремя или четырьмя джозефсоновскими контактами, критический ток одного из которых примерно на 20\% меньше критического тока остальных контактов. Это приводит к образованию двухуровневой системы с энергетической щелью, лежащей в гигагерцовой области частот. На данном этапе СПК является наиболее перспективным кандидатом в качестве элементной базы твердотельного квантового процессора. Здесь важно отметить, что, в отличие от обычных атомов, величиной энергетической щели СПК сравнительно легко управлять с помощью внешнего магнитного поля, приложенного к петле кубита. Другой важной особенностью, которая отличает СПК от обычных атомов, является то, что, вследствие технологического разброса параметров, СПК в принципе не идентичны, что следует учитывать при создании на их основе квантовых твердотельных метаматериалов [4].

\section{2. Описание эксперимента}

Сверхпроводниковый резонатор и сверхпроводниковый потоковый кубит были изготовлены в два техно- логических цикла в чистой комнате Лейбниц института фотонных технологий города Йена (Германия) технологией вакуумного напыления и технологией теневого напыления, соответственно. Толщина пленки резонатора материала $\mathrm{Nb}$ составила $200 \mathrm{~nm}$. Вся структура размещается на слаболегированной высокоомной кремниевой подложке и представляла собой резонатор в виде копланарной линии передачи [5], согласованной с волновым сопротивлением подводящих трактов $50 \Omega$. Три Al туннельных контакта Джозефсона, включенные в сверхпроводниковую петлю, формируют потоковый кубит, гальванически изолированный от сигнальных цепей копланарного резонатора. Структура кубита расположена в центральной зауженной части резонатора, где переменное электромагнитное поле имеет максимальную амплитуду магнитной составляющей (см. рис. 1 в [5]), что обеспечивает максимальную энергию взаимодействия кубита и резонатора.

Резонансная частота резонатора $f_{C}=2.59 \mathrm{GHz}$ выбрана из условия $f_{C}<\Delta$, где $\Delta-$ минимальная энергия щели кубита, соответствующая нулевой отстройке кубита (точке, при которой отсутствует сигнальный магнитный поток). Ширина ненагруженной кубитом резонансной линии оказалась равной $25 \mathrm{kHz}$, что соответствует добротности $Q \approx 10^{5}$. Длина резонатора, равная половине длины волны, составляла $23 \mathrm{~mm}$, ширина центрального волновода $50 \mu \mathrm{m}$, и зазор между заземлением и центральным проводником $30 \mu \mathrm{m}$.

Экспериментальное исследование сверхпроводниковой структуры резонатор-кубит осуществлялось в рефрижераторе растворения BF-LD400 при номинальной температуре $10 \mathrm{mK}$. Для уменьшения воздействия внешних электромагнитных помех использовался магнитный экран с коэффициентом подавления 100 для постоянного магнитного поля и более 8000 для переменного электромагнитного поля от частоты $10 \mathrm{~Hz}$ и выше. 
Во всех проведенных экспериментах использовался передаточный метод измерения. В узком спектре частот относительно $\omega_{C}$ в режиме малой мощности происходит измерение коэффициента передачи сверхпроводниковой цепи от частоты векторным анализатором цепей (ВАЦ). Из анализа комплексного коэффициента передачи были получены амплитуда и фаза выходного сигнала в зависимости от внешнего постоянного магнитного поля, прикладываемого через внешнюю катушку индуктивности, величиной порядка $1 \mathrm{mH}$. Для измерений кубита использовался уровень малой мощности сигнала накачки резонатора порядка $-140 \mathrm{dBm}\left(10^{-17} \mathrm{~W}\right)$. Сигнал малой мощности на выходе резонатора был усилен малошумящим криогенным усилителем.

\section{3. Методика определения параметров кубита}

СПК характеризуется четырьмя параметрами: энергетической щелью $\Delta$, незатухающим током в кольце интерферометра $I_{q}$, параметром релаксации $\gamma$ и энергией взаимодействия кубита с микроволновым трактом $g$. (Здесь и далее энергетические характеристики кубита $\Delta, g, \gamma$ приводятся в частотных единицах). Первые два параметра можно определить либо в дисперсионном режиме, когда резонансная частота сверхпроводникового резонатора $f_{C}=\omega_{C} / 2 \pi$ много меньше щели $\left(f_{C} \ll \Delta\right)[6-8]$, либо методом двухчастотной спектроскопии, когда в дополнение к пробному сигналу кубит возбуждается высокой частотой, близкой к расстоянию между энергетическими уровнями кубита [5]. Что касается двух других параметров, то $g$ обычно рассчитывается численно, исходя из дизайна структуры кубит-резонатор, а $\gamma$ определяется как подгоночный параметр для характерного провала в зависимости коэффициента прохождения от смещения кубита на фиксированной частоте [5].

Экспериментальное исследование свойств СПК, встроенного в микроволновой резонатор, производится путем измерения коэффициентов прохождения и/или отражения микроволнового сигнала, проходящего через такую структуру. Измеряя амплитуду и фазу проходящего сигнала $t=|t| \exp (i \varphi)$, мы определили параметры кубита и состояния, в которых он находится.

Аналитическое выражение для коэффициента прохождения микроволнового сигнала через копланарную линию, взаимодействующую посредством индуктивной связи со встроенным СПК, имеет следующий вид:

$$
t=\frac{-i \Gamma(\omega-\Omega+i \gamma)}{(\omega-\Omega+i \gamma)\left(\omega-\omega_{C}+i \Gamma\right)-\lambda^{2}},
$$

где $\omega-$ частота входного пробного сигнала, Г - полуширина резонансной линии микроволнового резонатора, $\gamma$ - диссипативные потери кубита, не связанные с излучением фотонов; $\omega_{C}-$ резонансная частота резонатора, $\Omega$ - энергия возбуждения кубита, $\Omega=\sqrt{\Delta^{2}+\varepsilon^{2}}$, $\varepsilon=2 I_{q} \delta \Phi_{\text {ext }} / \hbar, \delta \Phi_{\text {ext }}=\Phi_{x}-(n+1 / 2) \Phi_{0},(n=0,1,2 \ldots)$,
$I_{q}$ - незатухающий ток в кольце интерферометра, $\Phi_{0}=h / 2 e-$ квант магнитного потока, $\Phi_{x}-$ внешний магнитный поток, который представляет собой сумму паразитного захваченного потока $\Phi_{t}$ и потока $\Phi_{s}$, приложенного к интерферометру со стороны сигнальной катушки, $\Phi_{s}=M I_{s}$, где $M-$ взаимная индуктивность между кубитом и сигнальной катушкой, $I_{s}$ - ток в сигнальной катушке. Затухание населенности кубита в (1) описывается параметром $\gamma=\gamma_{1} / 2+\gamma_{\varphi}$, который включает в себя как чистую релаксацию $\gamma_{1}$, так и потери, связанные с декогерентностью $\gamma_{\varphi}$. Связь кубита с резонатором определяется параметром $\lambda=g \Delta / \Omega$, где $g$ - энергия взаимодействия кубита с основной модой резонатора.

Формула (1) впервые была получена в [9] при исследовании прохождения оптического излучения по оптоволокну, содержащему один двухуровневый атом, и независимо, и другим способом, была выведена в [10] применительно к распространению микроволнового сигнала в резонаторе, содержащем СПК. Впоследствии эта формула применялась в экспериментальной работе [5] для характеризации кубита при $\omega_{C}>\Delta$.

Выражение (1) справедливо в одномодовом и однофотонном приближениях: взаимодействие кубита с другими модами резонатора не учитывается, и в резонаторе не может быть больше одного фотона. Практически это означает, что среднее число фотонов в резонаторе должно быть меньше единицы. В нашем случае для среднего числа фотонов в резонаторе, соответствующего мощности пробного сигнала $P_{\text {in }}=10^{-17} \mathrm{~W}$, получим оценку $\langle N\rangle \approx 0.2$ (детали этого расчета приведены в приложении). Эту оценку следует сравнить со средним числом тепловых фотонов $\langle N\rangle \approx \exp \left(-h f_{C} / k_{B} T\right)$, которое для наших параметров $f_{C}=2.59 \mathrm{GHz}, T=10 \mathrm{mK}$ составляет величину порядка $10^{-6}$.

Зависимость амплитуды коэффициента прохождения $|t|$ от смещения $\varepsilon$ имеет характерный провал вблизи точки вырождения $(\varepsilon=0)$ [5]. Благодаря свойству периодичности состояний кубита во внешнем магнитном поле, этот провал повторяется при сдвиге внешнего магнитного потока $\Phi_{x}$ на целое число квантов магнитного потока $\Phi_{0}$ : $\left(\varepsilon=0\right.$ при $\left.\Phi_{x}=(n+1 / 2) \Phi_{0}\right)$. Это обстоятельство позволяет исключить паразитный магнитный поток $\Phi_{t}$ и определить взаимную индуктивность $M$. В нашем случае расстояние по сигнальному току $\Delta I_{S}$ между соседними провалами составило $1.54 \mathrm{~mA}$, откуда $M=\Phi_{0} / \Delta I_{S}=1.342 \mathrm{pH}$. Характерная форма такого провала показана на рис. 1. Каждая экспериментальная точка на этом графике соответствует точке на амплитудночастотной характеристике $(\mathrm{AЧX})$ на частоте $f_{C}$. Для каждой такой точки экспериментально получены соответствующие АЧХ коэффициента прохождения пробного сигнала.

На рис. 2 показаны две АЧХ, снятые для двух сигнальных токов, которым соответствуют точки $A$ и $B$ на рис. 1 . Точка $A$ соответствует току в сигнальной катушке $I_{s}=0.801 \mathrm{~mA}$. В этой точке влияние кубита минимально 


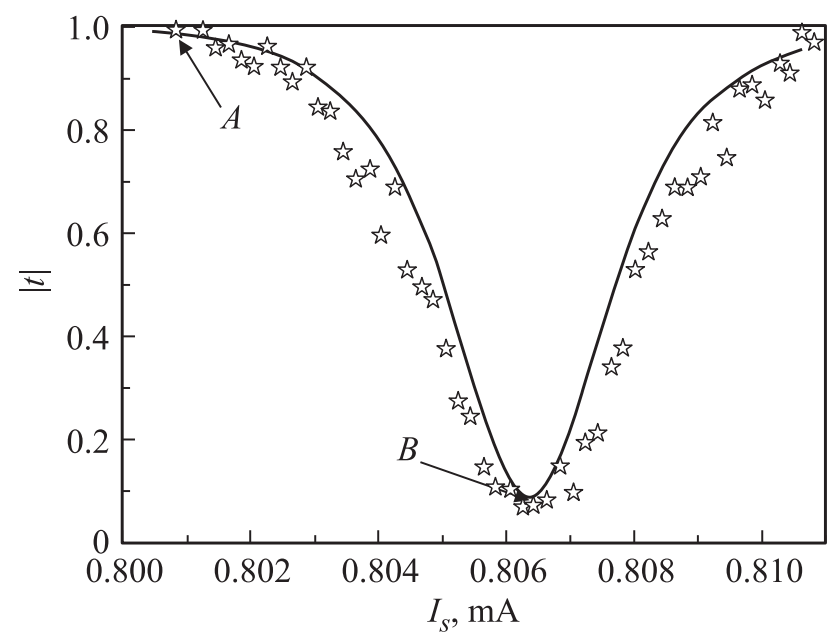

Рис. 1. Зависимость нормированной амплитуды коэффициента прохождения микроволнового сигнала на частоте резонанса $f_{C}=2.59 \mathrm{GHz}$ от тока смещения $I_{s}$ в сигнальной катушке. Экспериментальные точки, каждая из которых соответствует точке на АЧХ на частоте $f_{C}$, обозначены звездочками. Точки $A$ и $B$, АЧХ для которых представлены на рис. 2, соответствуют токам $0.801 \mathrm{~mA}$ и $0.8064 \mathrm{~mA}$ соответственно. Точка вырождения $(\varepsilon=0)$ соответствует сигнальному току $I_{s}=0.8064 \mathrm{~mA}$. Сплошная кривая рассчитана по формуле (1) для параметров, определенных из анализа частотного сдвига АЧХ и ее ширины: $\Delta=2.615 \mathrm{GHz}, I_{q}=180 \mathrm{nA}, g=2.63 \mathrm{MHz}, \gamma=4.73 \mathrm{MHz}$.

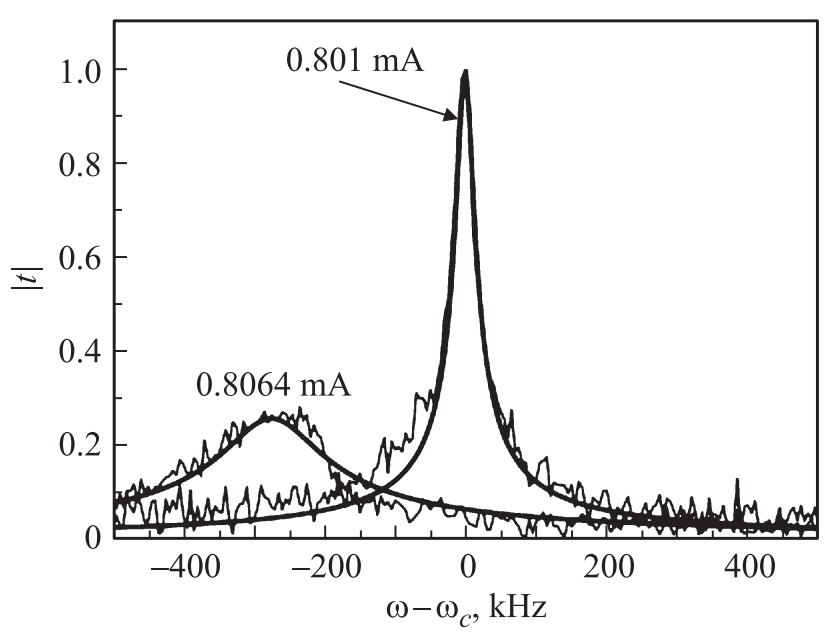

Рис. 2. Зависимость нормированного амплитудного значения коэффициента прохождения от частотной отстройки для двух значений сигнального тока. АЧХ для токов $0.801 \mathrm{~mA}$ и 0.8064 $\mathrm{mA}$ соответствуют точки $A$ и $B$ на рис. 1 соответственно. Сплошные линии рассчитаны по формуле (1) для параметров, определенных из анализа частотного сдвига АЧХ и ее ширины: $\Delta=2.615 \mathrm{GHz}, I_{q}=180 \mathrm{nA}, g=2.63 \mathrm{MHz}, \gamma=4.73 \mathrm{MHz}$.

и АЧХ практически совпадает с резонансной кривой резонатора. Точка $B$, для тока $I_{s}=0.8064 \mathrm{~mA}$, соответствует точке вырождения, в которой энергия возбуждения кубита минимальна. Вследствие влияния кубита, эта АЧХ смещена относительно фундаментальной частоты резонатора и существенно уширена. Как видно из рис. 2 , добротность сигнала в точке вырождения заметно ниже, что объясняется перекачиванием электромагнитной энергии возбужденным кубитом в моды, не связанные с основной модой резонатора.

Поскольку значения АЧХ в точках, лежащих на кривой провала (экспериментальные точки, показанные звездочками на рис. 1), значительно зашумлены, то использование для определения параметров кубита значения экспериментального коэффициента прохождения в этих точках довольно затруднительно. Поэтому в настоящей работе предлагается определять параметры кубита не по значениям амплитуды или фазы коэффициента прохождения на частоте $f_{C}$, а по величине смещения резонансной частоты АЧХ и ее ширине.

Взаимодействие кубита с резонатором приводит, вопервых, к сдвигу резонансной частоты АЧХ, и, вовторых, - к ее уширению (см. рис. 2). Положение резонансов в комплексной плоскости частоты $\omega_{c}$ определяется комплексными корнями знаменателя в (1). В случае, когда резонансная частота $\omega_{c}$ значительно отличается от энергии кубита $\Omega\left(\left|\omega_{c}-\Omega\right| \gg \Gamma, \gamma, g\right)$, положения этих корней с точностью до $g^{2}$ определяются следующими простыми выражениями:

$$
\begin{aligned}
& \omega_{+}=\omega_{c}+\frac{\lambda^{2}}{\omega_{c}-\Omega}-i\left(\Gamma+\frac{\lambda^{2} \gamma}{\left(\omega_{c}-\Omega\right)^{2}}\right), \\
& \omega_{-}=\Omega-\frac{\lambda^{2}}{\omega_{c}-\Omega}-i\left(\gamma-\frac{\lambda^{2} \gamma}{\left(\omega_{c}-\Omega\right)^{2}}\right) .
\end{aligned}
$$

Таким образом, при этих условиях смещение максимума АЧХ по частоте равно $\lambda^{2} /\left(\omega_{c}-\Omega\right)$, а дополнительное уширение АЧХ, связанное со взаимодействием кубита с резонатором, равно $\lambda^{2} \gamma /\left(\omega_{c}-\Omega\right)^{2}$. Положением резонанса и его шириной можно управлять, изменяя с помощью сигнального тока $I_{S}$ энергетическую щель кубита, которая определяется параметром $\Omega$. В точке вырождения (при $\varepsilon=0$ ) этот параметр принимает минимальное значение $(\Omega=\Delta)$.

В экспериментах регистрировались АЧХ на выходе сверхпроводникового резонатора вблизи его фундаментальной частоты. Анализ АЧХ проводился с помощью выражения (1) для коэффициента прохождения пробного сигнала. Из измерения смещения резонанса и ширины этих АЧХ были на основе выражений (2) и (3) определены такие параметры кубита, как его энергетическая щель, незатухающий ток в петле интерферометра, энергия связи кубита с резонатором, скорость его релаксации.

На рис. 3 и рис. 4 показаны зависимости частотного сдвига и ширины АЧХ от тока смещения в сигнальной катушке. Теоретические зависимости, рассчитанные по формуле (2), показаны сплошной линией. Наилучшее совпадение с экспериментально определенными значениями частотных сдвигов и ширин АЧХ, достигается при следующих параметрах кубита: $\gamma=4.73 \mathrm{MHz}$, $g=2.63 \mathrm{MHz}, \Delta=2.615 \mathrm{GHz}, I_{q}=180 \mathrm{nA}$. 


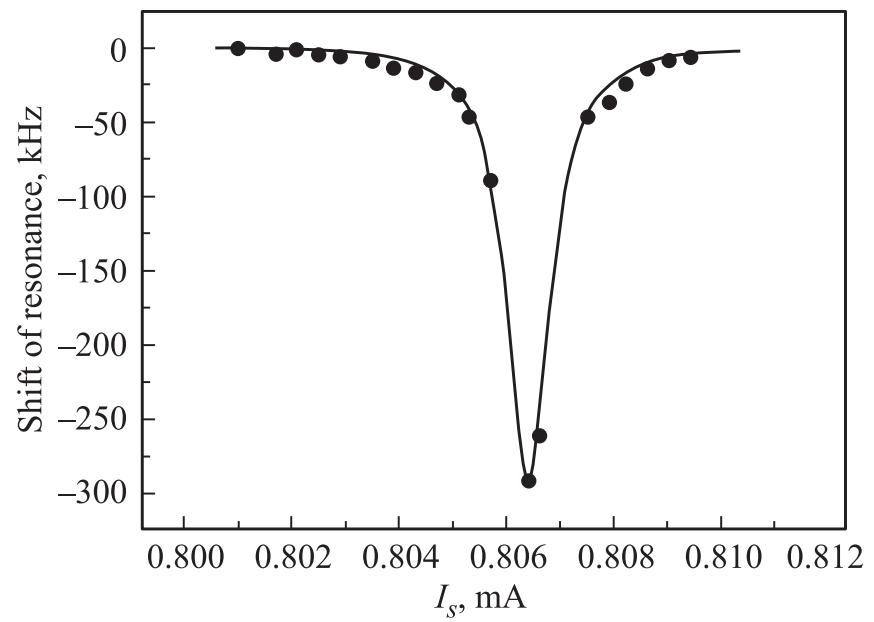

Рис. 3. Зависимость сдвига резонансной частоты АЧХ $\left(\omega-\omega_{C}\right)$ от тока в сигнальной катушке. Экспериментальные точки отмечены черными кружками. Сплошная линия представляет собой теоретическое смещение частоты $\lambda^{2} /\left(\omega_{C}-\Omega\right)$.

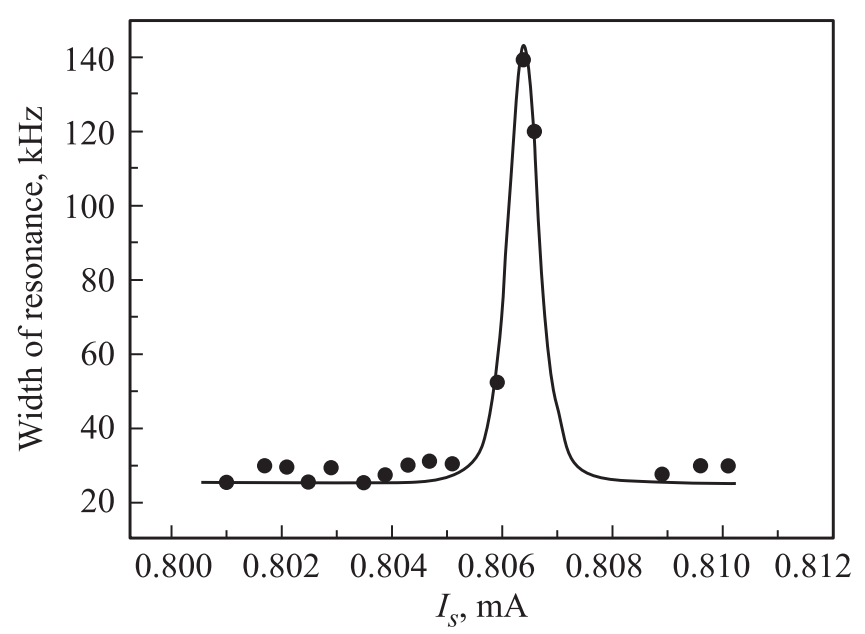

Рис. 4. Зависимость ширины АЧХ от тока в сигнальной катушке. Экспериментальные точки отмечены черными кружками. Сплошная линия представляет собой теоретическое значение ширины $2\left(\Gamma+\lambda^{2} \gamma /\left(\omega_{C}-\Omega\right)^{2}\right)$.

С помощью теоретических зависимостей для $\Delta$ и $I_{q}[11]$ мы нашли, что вышеуказанным значениям этих параметров соответствуют параметр несимметрии кубита $\alpha=0.678$ и отношение джозефсоновской и кулоновской энергий $E_{J} / E_{C}=57$.

\section{4. Заключение}

В работе описана новая экспериментальная методика определения параметров сверхпроводникового потокового кубита в квазидисперсионном режиме, когда частота микроволнового сигнала ненамного отличается от частоты возбуждения кубита. Методика не требует двухчастотного возбуждения кубита и основана на сравнении измеренных значений ширины и частотного смещения АЧХ с выражением (2), которое следует из теоретической формулы (1) для коэффициента прохождения.

\section{Приложение. \\ Оценка среднего равновесного числа инжектируемых фотонов в резонаторе}

Среднее число фотонов накачки в резонаторе связано с мнимой частью коэффициента прохождения [12] $\langle N\rangle=\Omega^{2} \operatorname{Im} t / \Delta f^{2}$, где $\Omega-$ энергия сигнала накачки в частотных единицах, $\Delta f$ - ширина полосы резонатора. В точке резонанса и при $\gamma \approx g \gg \Gamma$, что имеет место в нашем случае, выражение для среднего числа фотонов накачки можно привести к следующему виду $\langle N\rangle=\pi \Omega^{2} Q / \gamma \omega_{c}$, где $Q-$ добротность резонатора. Величина $\Omega$ зависит от конструктивных особенностей сверхпроводникового резонатора. Для используемой в данной работе структуры резонатора выражение для $\Omega$ имеет следующий вид [8]: $\Omega=C_{k} V_{\text {in }} V_{r} / h$, где $C_{k}-$ эквивалентная емкость зазора между входной частью копланарной линии передачи и центральным проводником, $V_{\text {in }}$ - амплитуда напряжения на входе резонатора $V_{\text {in }}(t)=V_{\text {in }} \cos (2 \pi f t)$, которая связана со входной мощностью $P_{\text {in }}=V_{\text {in }}^{2} / Z$, где в нашем случае $Z=50 \Omega$; $V_{r}$ - амплитуда напряжения сигнала внутри резонатора, $V_{r}=\left(h \omega_{c} / 2 \pi C_{r}\right)^{1 / 2}$, где $C_{r}-$ эквивалентная емкость резонатора. Таким образом, величины $\Omega$ и $\langle N\rangle$ можно записать через эти параметры следующим образом:

$$
\begin{gathered}
\Omega=C_{k} \sqrt{Z P_{\text {in }} \omega_{c} / 2 \pi h C_{r}}, \\
\langle N\rangle=C_{k}^{2} Z P_{\text {in }} Q / 2 \gamma h C_{r} .
\end{gathered}
$$

Для оценки мы взяли емкостной параметр $C_{k}=6 \mathrm{fF}$ из работы [5], где использовалась аналогичная конструкция резонатора. Величина $C_{r}$ вычислялась из геометрии резонатора в соответствии с формулой (3) из работы [13]: $C_{r}=2.3 \mathrm{pF}$. Мощность пробного сигнала на входе резонатора составляла $-140 \mathrm{dBm}\left(P_{\text {in }}=10^{-17} \mathrm{~W}\right)$, добротность резонатора составляла $Q=1 \cdot 10^{5}$. Для этих параметров непосредственный расчет по приведенным выше соотношениям дает для энергии сигнала накачки в резонаторе $\Omega \approx 260 \mathrm{kHz}$ и для числа фотонов накачки $\langle N\rangle \approx 0.2$.

\section{Список литературы}

[1] I. Buluta, S. Ashhab, F. Nori. Rep. Prog. Phys. 74, 104401 (2011).

[2] O. Astafiev, A.M. Zagoskin, A.A. Abdumalikov, Yu.A. Pashkin, T. Yamamoto, K. Inomata, Y. Nakamura, J.S. Tsai. Science 327, 840 (2010).

[3] T.P. Orlando, J.E. Mooij, Lin Tian, C.H. van der Wal, L.S. Levitov, S. Lloyd, J.J. Mazo. Phys. Rev. B 60, 15398 (1999). 
[4] P. Macha, G. Oelsner, J.-M. Reiner, M. Marthaler, S. André, G. Schön, U. Hübner, H.-G. Meyer, E. Il'ichev, A.V. Ustinov. Nature Commun. 5, 5146 (2004).

[5] G. Oelsner, S.H.W. van der Ploeg, P. Macha, U. Hübner, D. Born, S. Anders, E. Il'ichev, H.-G. Meyer, M. Grajcar, S. Wünsch, M. Siegel, A.N. Omelyanchouk, O. Astafiev. Phys. Rev. B 81, 172505 (2010).

[6] M. Grajcar, A. Izmalkov, E. Il'ichev, Th. Wagner, N. Oukhanski, U. Hübner, T. May, I. Zhilyaev, H.E. Hoenig, Ya.S. Greenberg, V.I. Shnyrkov, D. Born, W. Krech, H.-G. Meyer, Alec Maassen van den Brink, M.H.S. Amin. Phys. Rev. B 69, 060501(R) (2004).

[7] M. Grajcar, A. Izmalkov, E. Il'ichev. Phys. Rev. B 71, 144501 (2005).

[8] V.I. Shnyrkov, Th. Wagner, D. Born, S.N. Shevchenko, W. Krech, A.N. Omelyanchouk, E. Il'ichev, H.-G. Meyer. Phys. Rev. B 73, 024506 (2006).

[9] J.-T. Shen, S. Fan. Phys. Rev. A 79, 023837 (2009).

[10] A.N. Omelyanchouk, S.N. Shevchenko, Ya.S. Greenberg, O. Astafiev, E. Il'ichev. Fizika Nizkikh Temperatur 36, 1117 (2010).

[11] Ya.S. Greenberg, A. Izmalkov, M. Grajcar, E. Il'ichev, W. Krech, H.-G. Meyer, M.H.S. Amin, Alec Maassen van den Brink. Phys. Rev. B 66, 214525( 2002).

[12] S.N. Shevchenko, G. Oelsner, Ya.S. Greenberg, P. Macha, D.S. Karpov, M. Grajcar, U. Hübner, A.N. Omelyanchouk, E. Il'ichev. Phys. Rev. B 89, 184504 (2014).

[13] M. Goppl, A. Fragner, M. Baur, R. Bianchetti, S. Filipp, J.M. Fink, P.J. Leek, G. Puebla, L. Steffen, A. Wallraff. J. Appl. Phys. 104, 113904 (2008). 